\title{
PARP-1 may be involved in angiogenesis in epithelial ovarian cancer
}

\author{
WEI WEI ${ }^{*}$, YAN LI* ${ }^{*}$ SHUQING LV, CANCAN ZHANG and YONGJIE TIAN \\ Department of Obstetrics and Gynecology, Provincial Hospital Affiliated to Shandong University, \\ Jinan, Shandong 250021, P.R. China
}

Received July 11, 2015; Accepted September 15, 2016

DOI: $10.3892 / \mathrm{ol} .2016 .5226$

\begin{abstract}
Poly (ADP-ribose) polymerase 1 (PARP-1) is involved in DNA repair and has been implicated in chemoresistance. The present study investigated whether PARP-1 promotes angiogenesis in ovarian cancer. PARP-1 and vascular endothelial growth factor A (VEGF-A) expression and $\mathrm{CD}_{3} 4^{+}$microvascular density (MVD) were assessed using immunohistochemistry in 60 human epithelial ovarian cancer specimens. PARP-1 was stably knocked-down in SKOV3 cells using a specific small interfering RNA (siRNA); angiogenic capacity was assessed using the human umbilical vein endothelial cell (HUVEC) tubule formation assay; and PARP-1 and VEGF-A expression were examined by reverse transcription-quantitative polymerase chain reaction, western blotting and ELISA. PARP-1 was found to be expressed in $73.3 \%$ (44/60) of the human epithelial ovarian cancer specimens and was significantly associated with VEGF-A, MVD, tumor size, histological grade and lymphatic metastasis $(\mathrm{P}<0.05)$. Compared with cells transfected with a negative control siRNA, knockdown of PARP-1 significantly suppressed the ability of SKOV3 cell-conditioned media to promote HUVEC tubule formation on Matrigel in vitro. Knockdown of PARP-1 in SKOV3 cells also significantly reduced VEGF-A mRNA and protein expression and secretion. In summary, PARP-1 is overexpressed and may enhance angiogenesis in epithelial ovarian cancer by upregulating VEGF-A.
\end{abstract}

\section{Introduction}

Epithelial ovarian cancer is highly invasive and has the highest mortality rate among the various types of gynecological malignancy (1). At the time of diagnosis, $>70 \%$ of patients have

Correspondence to: Professor Yongjie Tian, Department of Obstetrics and Gynecology, Provincial Hospital Affiliated to Shandong University, 324 Jingwu Road, Jinan, Shandong 250021, P.R. China

E-mail: tianyongjie@sdu.edu.cn

*Contributed equally

Key words: ovarian cancer, poly (ADP-ribose) polymerase 1, siRNA, angiogenesis, vascular endothelial growth factor A advanced stage disease (1). Currently, the standard treatment is cytoreductive surgery followed by platinum-and paclitaxel-based chemotherapy. However, the efficacy of chemotherapy is challenged by chemoresistance and tumor recurrence (2). Therefore, molecular targeted therapy for ovarian cancer has become a novel field of research in recent years (3). Existing molecular targeting agents are predominantly monoclonal antibodies that target proteins that are abnormally expressed in tumor cells, or small molecule protein kinase inhibitors that regulate cell growth or inhibit angiogenesis; these agents have a higher specificity and lower toxicity compared with traditional chemotherapeutic agents (4).

Poly (ADP-ribose) polymerase 1 (PARP-1) is expressed in the nuclei of most eukaryotic cells and participates in DNA damage repair, gene transcription, the cell cycle, chromosome function, genomic stability and cell death (5). PARP-1 contains three structural domains: A DNA binding domain, an auto-modification domain and a catalytic domain (6). Once activated by DNA damage, PARP-1 rapidly forms homodimers that recognize and bind DNA nicks, whereupon it catalyzes ADP ribosylation of itself and histones (7), leading to chromosomal relaxation and thereby recruitment of DNA polymerase $\beta$, X-ray repair cross-complementing protein 1 and DNA ligase III to sites of DNA damage to initiate DNA repair (8). When DNA damage is severe, PARP-1 becomes over-activated, which can lead to the depletion of $\mathrm{NAD}^{+}$and ATP and subsequently induce cell death (9).

Our previous research demonstrated that PARP-1 inhibitors can enhance the chemosensitivity of ovarian cancer cells in vitro (10). Notably, the PARP-1 inhibitor PJ34 can also inhibit angiogenesis in the chorioallantoic membrane assay (11). In order to investigate whether PARP-1 may be involved in angiogenesis in ovarian cancer, the present study examined the expression of PARP-1 and its association with markers of angiogenesis in human epithelial ovarian cancer. Furthermore, the effect of PARP-1 on the angiogenic capacity of ovarian cancer cells in vitro was investigated.

\section{Materials and methods}

Patients and samples. Tissue samples from 60 patients with epithelial ovarian cancer treated at the Department of Gynecology, Provincial Hospital Affiliated to Shandong University (Jinan, China) between January 2013 and June 2014 were used. 
The mean age of the patients was 58 years (range, 38-77 years). No patients had a prior history of chemotherapy, radiotherapy or immunotherapy. All patients were staged at the time of surgery according to the International Federation of Gynecology and Obstetrics (FIGO) staging guidelines (FIGO 2000). The tissues were collected from patients after obtaining informed consent from the patients' families. The study was approved by the ethics committee of the Provincial Hospital Affiliated to Shandong University.

Immunohistochemistry. Formalin-fixed, paraffin-embedded epithelial ovarian cancer tissues were sectioned $(4 \mu \mathrm{m})$, deparaffinized, and incubated with $3 \%$ hydrogen peroxide followed by rabbit monoclonal anti-PARP-1 (Cell Signaling Technology, Inc., Danvers, MA, USA; 9532S; dilution, 1:1,000), mouse monoclonal anti-VEGF-A (Santa Cruz Biotechnology, Inc., Santa Cruz, CA, USA; sc-57496; dilution, 1:50) or mouse monoclonal anti-CD34 (a marker for vascular endothelial cells) (Santa Cruz Biotechnology, Inc.; sc-19621; dilution, 1:100) at $4^{\circ} \mathrm{C}$ overnight. Subsequently, the sections were processed using a secondary biotinylated antibody kit (SP-9000 detection kit; OriGene Technologies, Inc., Beijing, China), following the manufacturer's instructions. For negative controls, primary antibody was replaced with PBS.

All sections were examined by two independent pathologists who were blinded to the clinical data. PARP-1 was predominantly localized to the tumor cell nuclei. Samples in which $>10 \%$ of cells were positive were considered PARP-1-positive. A VEGF-A staining score was calculated by multiplying the score for the percentage of positive cells $(0$, no positive tumor cells; $1, \leq 25 \%$ positive tumor cells; $2,26-50 \%$ positive tumor cells; $3,51-75 \%$ positive tumor cells; and $4, \geq 76 \%$ positive tumor cells) by the staining intensity score ( 0 , negative; 1 , weak; 2 , moderate; 3 , strong). A score of 0-3 was considered to indicate low expression and a score of $\geq 4$ was considered to indicate high expression.

Measurement of microvessel density (MVD). MVD was measured by assessing the CD34-positive vessels in five fields of view. In each case, the most vascularized area was selected and the microvessels within a high-power magnification (x200) field of vision were counted three times. Macrovascular structures with smooth muscle cells were excluded. The mean of the three highest counts per tumor was used for analysis.

Knockdown of PARP-1. SKOV3 human ovarian cancer cells and human umbilical vein endothelial cells (HUVECs) were obtained from the Central Laboratory of the Provincial Hospital Affiliated to Shandong University, and were cultured in Hyclone RPMI-1640 medium (GE Healthcare Life Sciences, Logan, UT, USA) supplemented with $10 \%$ fetal bovine serum (FBS) (Hyclone; GE Healthcare Life Sciences) at $37^{\circ} \mathrm{C}$ in a humidified incubator with 5\% $\mathrm{CO}_{2}$. A small interfering RNA (siRNA) (5'-AAGATAGAGCGTGAAGGCGAA-3') that specifically targets PARP-1 (GenBank accession number, NM_001618) and negative control (NC) siRNA that does not target any known human gene (5'-TTCTCCGAACGTGTCACGT-3') were designed and inserted into lentiviral vectors (pGCL green fluorescent protein vector) by Shanghai Genechem Co., Ltd. (Shanghai, China).
SKOV3 cells $\left(\sim 5 \times 10^{4}\right)$ were seeded into 12 -well plates, and cultured for $24 \mathrm{~h}$ in RPMI-1640 medium supplemented with $10 \%$ FBS. The media was then replaced with $500 \mu \mathrm{l}$ of suspension solution containing $5 \mu \mathrm{g} / \mathrm{ml}$ polybrene, $250 \mu 1$ Enhanced Infection Solution (GeneChem Co., Ltd.) and the lentiviral constructs $(25 \mu \mathrm{l})$. After $16 \mathrm{~h}$, the suspension was replaced with complete medium containing puromycin $(1 \mu \mathrm{g} / \mathrm{ml})$ to select stably transfected cells.

HUVEC tubule formation assay. Matrigel (BD Biosciences, Bedford, MA, USA) was added to 96-well culture plates (60 $\mu \mathrm{l} /$ well) and allowed to polymerize at $37^{\circ} \mathrm{C}$ for $30 \mathrm{~min}$. Conditioned media was collected from SKOV3 cells transfected with NC-siRNA or PARP-1-siRNA, and was centrifuged for $5 \mathrm{~min}$ at $1,000 \mathrm{x} g$ at room temperature to remove cells. HUVECs were resuspended in the conditioned media $\left(2.5 \times 10^{5}\right.$ cells $\left./ \mathrm{ml}\right)$, and 100- $\mu \mathrm{l}$ aliquots were seeded onto the Matrigel, incubated at $37^{\circ} \mathrm{C}$ for $18 \mathrm{~h}$, and imaged using an inverted phase contrast microscope.

Reverse transcription-quantitative polymerase chain reaction (RT-qPCR). Total RNA was isolated from SKOV3 cells using Invitrogen TRIzol reagent (Thermo Fisher Scientific, Inc., Carlsbad, CA, USA) according to the manufacturer's protocol. The purified RNA was suspended in diethyl pyrocarbonate-treated water. Total RNA (10 $\mu \mathrm{l})$ obtained from each of the cell cultures was converted into cDNA using oligo-dT15 primers and M-MLV reverse transcriptase (Promega Corporation, Madison, WI, USA). Primers specific to PARP-1 (forward, 5'-GCCCTAAAGGCTCAGAACGAC-3', and reverse, 5'-CAC CATGCCATCAGCTACTCG-3'), VEGF-A (forward, 5'-TCG AGACCCTGGTGGACATC-3', and reverse, 5'-CTATGTGCT GGCCTTGGTGAG-3') and $\beta$-actin (forward, 5'-AGCGAG CATCCCCCAAAGTT-3', and reverse, 5'-GGGCACGAAGGC TCATCATT-3') were designed and synthesized by Sangon Biotech Co., Ltd. (Shanghai, China). qPCR was performed in $20-\mu 1$ reaction mixtures containing $10 \mu$ l SYBR Green Realtime PCR Master Mix (Toyobo, Osaka, Japan), $4 \mu 1$ of each forward and reverse primer $(10 \mu \mathrm{mol} / \mathrm{l})$ and $2 \mu \mathrm{l}$ cDNA in a Lightcycler 2.0 (Roche Diagnostics, Indianapolis, IN, USA). The thermal conditions were $5 \mathrm{~min}$ at $95^{\circ} \mathrm{C}$, followed by 45 cycles of $5 \mathrm{~min}$ at $95^{\circ} \mathrm{C}, 10 \mathrm{sec}$ at $60^{\circ} \mathrm{C}$ and $10 \mathrm{sec}$ at $72^{\circ} \mathrm{C}$. The expression levels of $P A R P-1$ and $V E G F-A$ were normalized to that of $\beta$-actin using the $2^{-\Delta \Delta \mathrm{Cq}}$ method (12).

Western blotting and ELISA. Total cellular protein was extracted and protein concentrations were determined using Protein Assay Dye Reagent (Bio-Rad Laboratories, Inc., Cambridge, MA, USA). Subsequently, equal amounts of total cellular protein extracts were separated using $10 \%$ SDS polyacrylamide gel electrophoresis and transferred to a nitrocellulose membrane (Pierce; Thermo Fisher Scientific, Inc., Rockford, IL, USA). Membranes were incubated for $2 \mathrm{~h}$ in TBS containing $0.1 \%$ Tween-20 and 5\% bovine serum albumin to block non-specific binding. Membranes were then incubated overnight with primary antibodies at $4{ }^{\circ} \mathrm{C}$, and then with the appropriate secondary antibodies for $2 \mathrm{~h}$ at room temperature. The primary antibodies used were against PARP-1 (9532S, Cell Signaling Technology, Inc.; dilution, 1:1,000), VEGF-A (sc-57496, Santa Cruz Biotechnology, Inc.; dilution, 1:300) and $\beta$-actin (sc-47778, Santa Cruz 
A

$$
\text { PARP-1 positive group }
$$
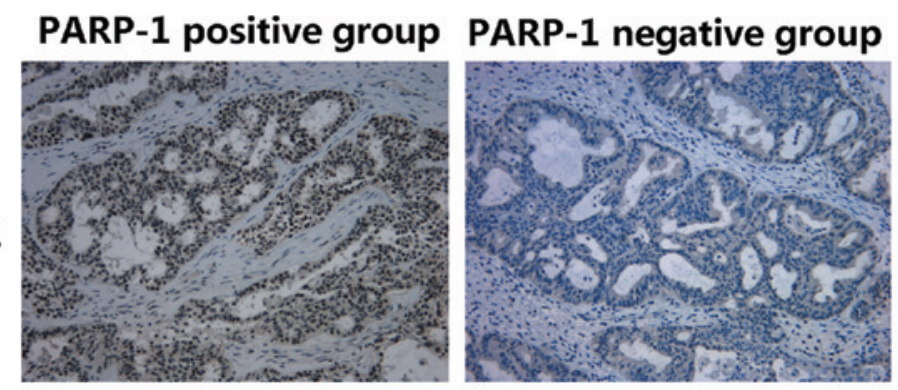

PARP-1
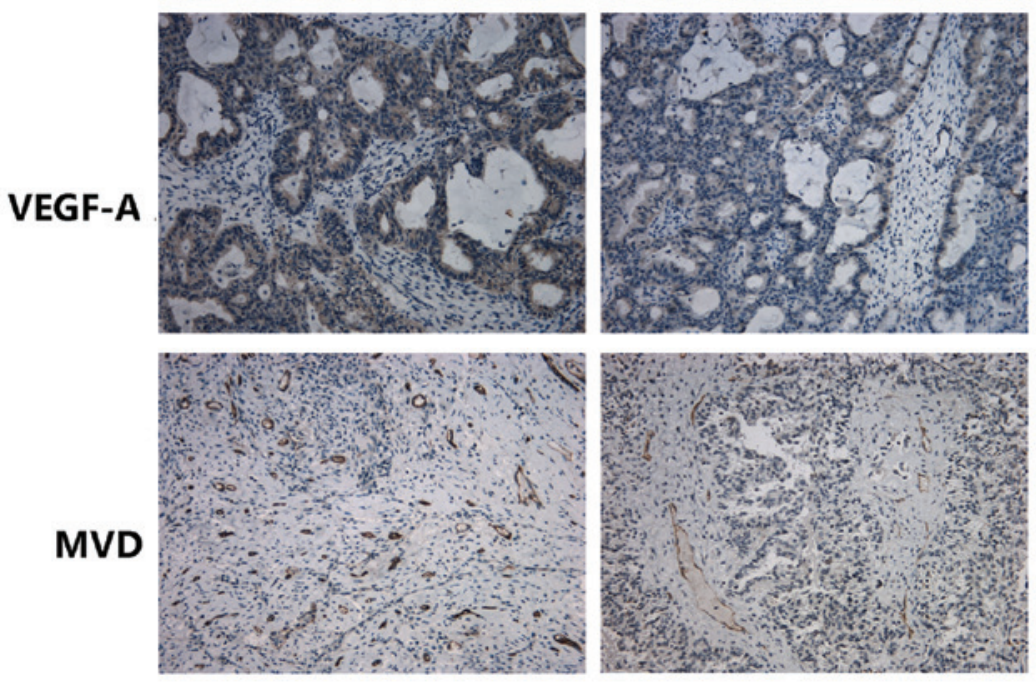

B

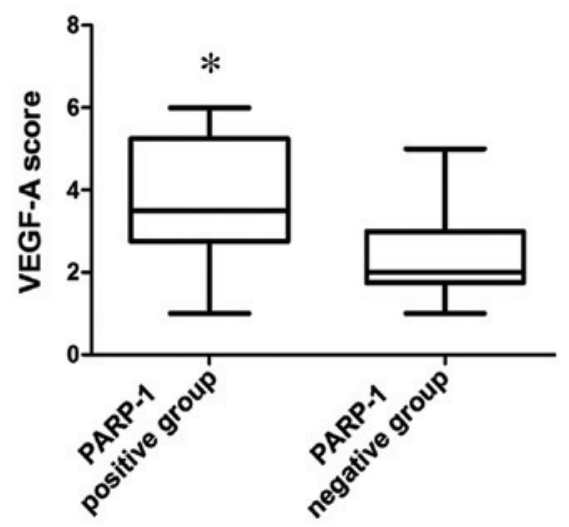

C

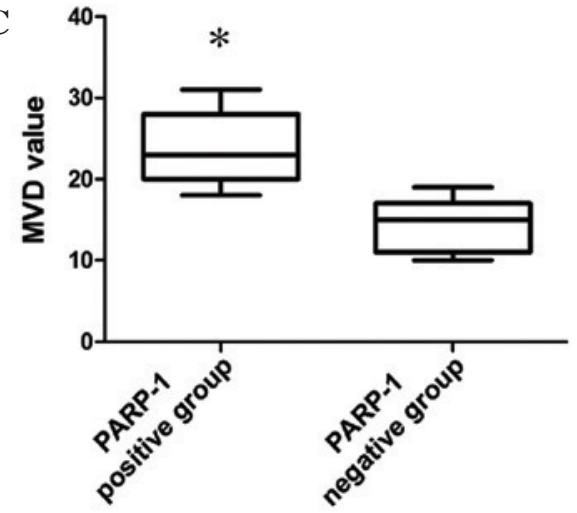

Figure 1. Association of PARP-1 with VEGF-A and MVD in human epithelial ovarian cancer. (A) Representative images of PARP-1, VEGF-A and CD34 (used to determine MVD) immunohistochemistry in PARP-1-positive and PARP-1-negative human ovarian cancer tissues (magnification, x200). (B) VEGF-A immunostaining score and (C) MVD values (per field) of PARP-1-positive $(\mathrm{n}=44)$ and PARP-1-negative $(\mathrm{n}=16)$ human ovarian cancer tissues. Data are presented as the mean \pm standard deviation of three independent experiments. "P<0.05. PARP-1, poly (ADP-ribose) polymerase 1; VEGF-A, vascular endothelial growth factor A; MVD, microvessel density.

Biotechnology, Inc.; dilution, 1:1,000). The secondary antibodies used were as follows: Horseradish peroxidase-conjugated goat anti-rabbit immunoglobulin (Ig)G (Santa Cruz Biotechnology, Inc.; sc-2004; dilution, 1:8,000) and goat anti-mouse IgG (Santa Cruz Biotechnology, Inc.; sc-2005; dilution, 1:8,000). Signals were detected using a Pierce enhanced chemiluminescence kit (Thermo Fisher Scientific, Inc.), and protein expression levels were quantified using Gel-Pro Analyzer software v6.0 (Media Cybernetics, Inc., Rockville, MD, USA). Results were normalized to the $\beta$-actin content in the samples.

The VEGF-A content of cell supernatants was measured using a VEGF-A ELISA (R\&D Systems, Inc., Minneapolis, MN, USA) following the manufacturer's instructions.

Statistical analysis. All in vitro experiments were performed in triplicate. Data were analyzed using SPSS software version 19.0 (IBM SPSS, Armonk, NY, USA). The associations between PARP-1 and clinicopathological features were assessed using the $\chi^{2}$ test or Fisher's exact probability test. The Student's $t$-test was used to compare experimental groups. $\mathrm{P}<0.05$ was considered to indicate a statistically significant difference and all data are expressed as the mean \pm standard error of the mean.

\section{Results}

Expression of PARP-1 and association with VEGF-A, MVD and clinicopathological features of human ovarian cancer.
The rate of positive PARP-1 staining in the human ovarian cancer specimens was $73.3 \%$ (44/60). VEGF-A was expressed in all 60 samples (mean score, $3.05 \pm 1.61$; range, 1-6) and the mean MVD was $19.14 \pm 6.24$ per field (Fig. 1A).

The mean VEGF-A staining score was significantly higher for patients with PARP-1-positive tumors $(3.80 \pm 1.69)$ compared with those with PARP-1-negative tumors (2.30 \pm 1.16 ; $P=0.026$; Fig. 1B). Additionally, patients with PARP-1-positive tumors had a significantly higher MVD (23.86 \pm 4.67 per field) than patients with PARP-1-negative tumors (14.43 \pm 3.26 per field; $P=0.01$; Fig. $1 C$ ).

Positive expression of PARP-1 was significantly associated with tumor size $(\mathrm{P}=0.018)$, histological grade $(\mathrm{P}=0.001)$ and lymphatic metastasis $(\mathrm{P}=0.005)$, but not age $(\mathrm{P}=0.464)$ or FIGO stage $(\mathrm{P}=0.302)$ in the 60 cases of human ovarian cancer (Table I).

Silencing of PARP-1 reduces the angiogenic capacity of SKOV3 cells and downregulates VEGF-A. RT-qPCR and western blot analyses confirmed that $P A R P I$-siRNA-transfected SKOV3 cells expressed significantly lower levels of PARP-1 than cells transfected with the NC-siRNA (Fig. 2).

HUVECs cultured on Matrigel in conditioned media from SKOV3 cells transfected with PARP-1-siRNA formed significantly fewer tubules than HUVECs cultured in conditioned media from cells transfected with NC-siRNA $(14.67 \pm 1.21$ vs. $8.83 \pm 1.47$ per high-power field; $\mathrm{P}=0.005$; Fig. 3 ), suggesting 
Table I. Association between PARP-1 and the clinicopathological features of human epithelial ovarian cancer.

Number of patients

\begin{tabular}{|c|c|c|c|c|c|c|}
\hline & & & & & & \\
\hline Feature & All & PARP-1-positive & PARP-1-negative & Positive rate (\%) & $\chi^{2}$ & P-value \\
\hline Age (years) & & & & & 0.536 & 0.464 \\
\hline$<50$ & 13 & 8 & 5 & 61.5 & & \\
\hline$\geq 50$ & 47 & 36 & 11 & 76.6 & & \\
\hline Tumor size $(\mathrm{cm})$ & & & & & 5.556 & 0.018 \\
\hline$<2$ & 18 & 9 & 9 & 50.0 & & \\
\hline$\geq 2$ & 42 & 35 & 7 & 83.3 & & \\
\hline Histological grade & & & & & 10.484 & 0.001 \\
\hline $\mathrm{G} 1+\mathrm{G} 2$ & 28 & 15 & 13 & 53.6 & & \\
\hline G3 & 32 & 29 & 3 & 90.6 & & \\
\hline FIGO stage & & & & & 1.065 & 0.302 \\
\hline $\mathrm{I}+\mathrm{II}$ & 20 & 13 & 7 & 65.0 & & \\
\hline $\mathrm{III}+\mathrm{IV}$ & 40 & 31 & 9 & 77.5 & & \\
\hline Lymphatic metastasis & & & & & 7.934 & 0.005 \\
\hline No & 27 & 15 & 12 & 55.6 & & \\
\hline Yes & 33 & 29 & 4 & 87.9 & & \\
\hline
\end{tabular}

PARP-1, poly (ADP-ribose) polymerase 1; FIGO, International Federation of Gynecology and Obstetrics.

A
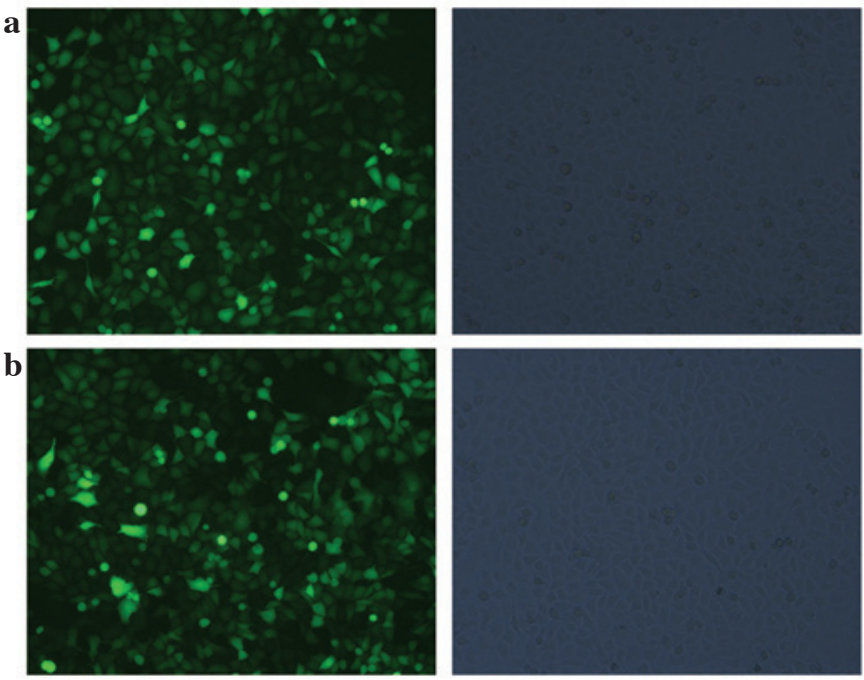

C

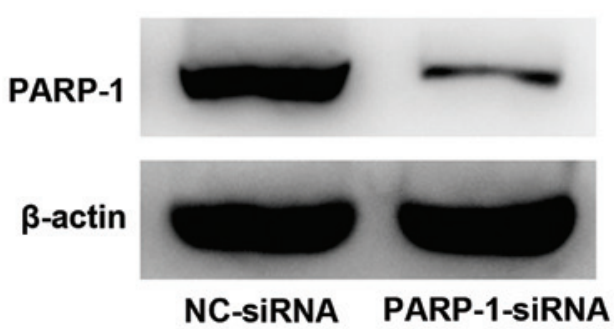

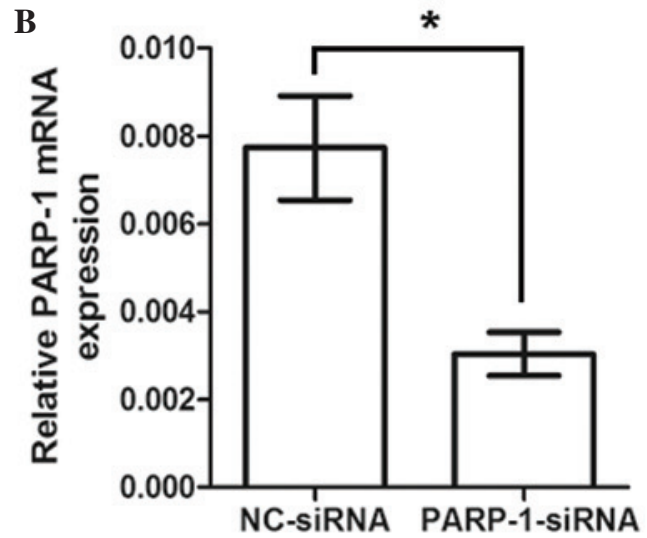

D

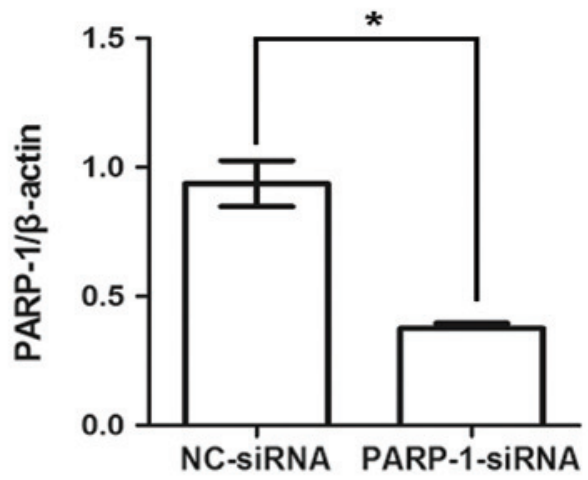

Figure 2. Knockdown of PARP-1 by siRNA. (A) Representative fluorescence and bright-field micrographs of SKOV3 cells transfected with lentiviruses expressing GFP and PARP-1-siRNA or NC-siRNA show that cells began to express GFP $12 \mathrm{~h}$ after transfection. The efficiencies of transfection were $89 \%$ and $86 \%$ for (a) NC-siRNA and (b) PARP-1-siRNA, respectively. (B) Reverse transcription-quantitative polymerase chain reaction and (C) western blot analyses of PARP-1 in SKOV3 cells transfected with PARP1-siRNA or NC-siRNA confirmed the knockdown of PARP-1 expression in PARP-1-siRNA-transfected cells. (D) Quantification of western blot analysis. Values are presented as the mean \pm standard deviation of three independent experiments; ${ }^{*}<0.05$. PARP-1, poly (ADP-ribose) polymerase 1; siRNA, small interfering RNA; GFP, green fluorescent protein; PARP-1-siRNA, siRNA targeting PARP-1; NC-siRNA, negative control siRNA. 


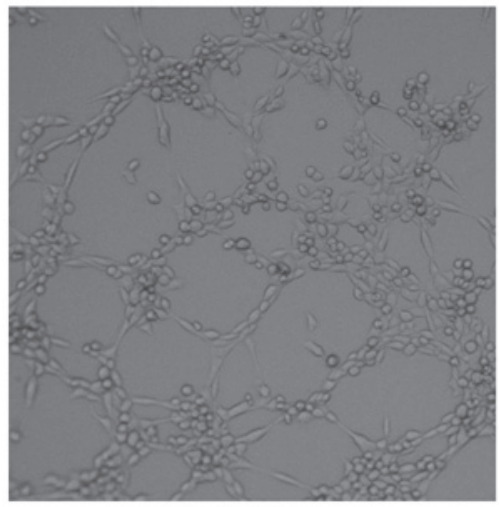

NC-siRNA

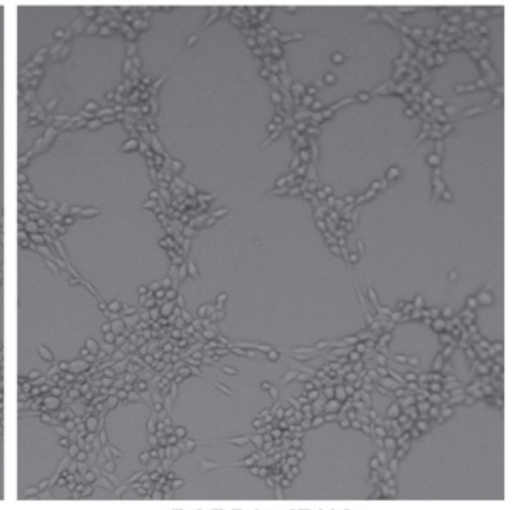

PARP1-SIRNA

Figure 3. Silencing of PARP-1 reduces the angiogenic capacity of SKOV3 cells in vitro. Representative images (magnification, x100) show tubule formation by human umbilical vein endothelial cells plated on Matrigel and cultured in conditioned media from SKOV3 cells transfected with PARPI-siRNA or NC-siRNA. PARP-1, poly (ADP-ribose) polymerase 1; siRNA, small interfering RNA; PARP-1-siRNA, siRNA targeting PARP-1; NC-siRNA, negative control siRNA.

A

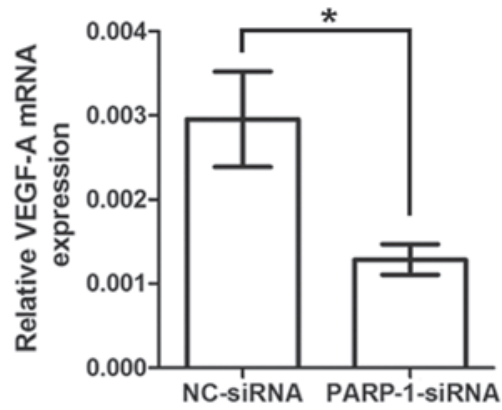

C

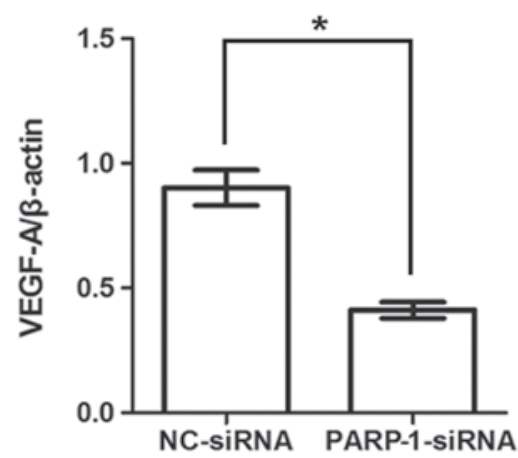

B

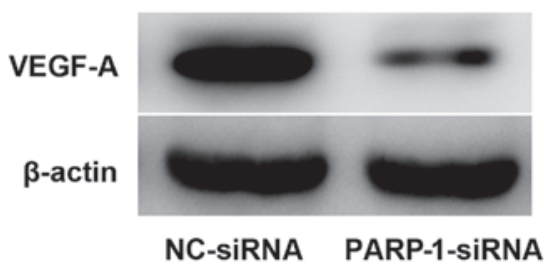

D

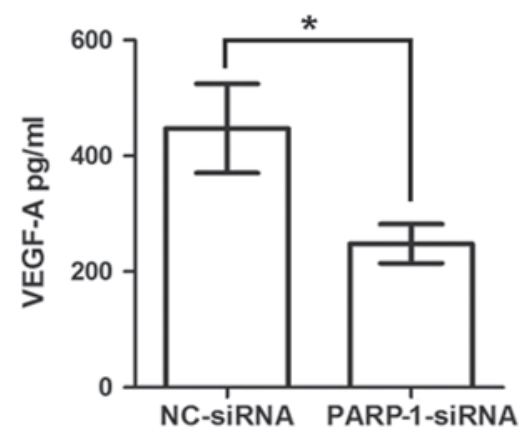

Figure 4. Silencing of PARP-1 downregulates VEGF-A in SKOV3 ovarian cancer cells. (A) Reverse transcription-quantitative polymerase chain reaction and (B) western blot analyses indicated reduced VEGF-A levels in SKOV3 cells transfected with PARPI-siRNA compared with NC-siRNA-transfected cells; (C) quantified western blot analysis results are shown. (D) ELISA of VEGF-A in the supernatant of SKOV3 cells expressing PARP-1-siRNA or NC-siRNA revealed reduced VEGF-A levels in the former group. Values are presented as the mean \pm standard deviation of three independent experiments; "P<0.05. PARP-1, poly (ADP-ribose) polymerase 1; VEGF-A, vascular endothelial growth factor A; siRNA, small interfering RNA; PARP-1-siRNA, siRNA targeting PARP-1; NC-siRNA, negative control siRNA.

that PARP-1 may enhance the ability of ovarian cancer cells to promote angiogenesis.

To investigate the mechanism by which PARP-1 affects the ability of ovarian cancer cells to promote endothelial cell tubule formation, the expression levels of VEGF-A in SKOV3 cells transfected with PARP-1-siRNA or NC-siRNA were examined. RT-qPCR demonstrated that SKOV3 cells transfected with $P A R P$-1-siRNA expressed lower levels of VEGF-A mRNA compared with cells transfected with NC-siRNA. Western blotting demonstrated that knockdown of PARP-1 significantly reduced the relative VEGF-A protein expression in SKOV3 cells $(0.41 \pm 0.08$ vs. $0.90 \pm 0.18$ for NC-siRNA; $\mathrm{P}=0.008$; Fig. 4B and $\mathrm{C}$ ). The level of VEGF-A in the cell supernatant was also reduced in $P A R P$-1-knockdown cells compared with the NC-siRNA-transfected cells (248.12 \pm 82.74 vs. $447.22 \pm 188.52 \mathrm{pg} / \mathrm{ml}$; Fig. 4D), as demonstrated by ELISA. Collectively, these results indicate $P A R P-1$ may upregulate VEGF-A in ovarian epithelial cancer cells.

\section{Discussion}

PARP-1 is overexpressed and serves important roles in the progression of breast cancer (13), prostate cancer (14) and pancreatic cancer (15). Additionally, PARP-1 has been associated with tumor invasion and lymph node metastasis in gastric cancer (16), and its overexpression associated with tumor 
stage, overall survival and prognosis in breast cancer (17). Indeed, PARP-1 is currently being investigated as a target for cancer therapy and a number of PARP-1 inhibitors have been tested in phase II clinical studies (18). In the present study, PARP-1 was found to be overexpressed in $73.3 \%(44 / 60)$ of the human epithelial ovarian cancer tissues examined, and was associated with tumor size, pathological grade and lymph node metastasis. These data indicate that PARP-1 may also be involved in the progression of ovarian cancer.

Angiogenesis is necessary for continued tumor growth and is a prerequisite for tumor invasion and metastasis. A high MVD is associated with poor prognosis in a range of tumor types, including esophageal (19), breast (20) and ovarian cancer (21). An association between PARP-1 and angiogenesis has also been reported in other types of cancer. For example, PARP-1 was found to be associated with MVD, and overexpression of PARP-1 enhanced the angiogenic capacity of colon cancer cells (22). Additionally, the PARP inhibitor DPQ was demonstrated to significantly inhibit the growth of human hepatocellular carcinoma xenografts in nude mice and attenuate angiogenesis during tumor progression via a process involving altered gene expression (23). In the present study, an association between PARP-1 and the MVD in human epithelial ovarian cancer was identified, indicating that PARP-1 exerts a pro-angiogenic effect in epithelial ovarian cancer. Additionally, knockdown of $P A R P-1$ significantly suppressed the ability of conditioned media from SKOV3 cells to promote HUVEC tubule formation in vitro, suggesting that PARP-1 may promote angiogenesis in ovarian cancer.

VEGF-A is a well-characterized pro-angiogenic factor that activates multiple downstream effectors, including extracellular signal-regulated kinases, Src and phosphoinositide 3-kinase/Akt, to stimulate endothelial cell proliferation, invasion and basement membrane degradation (24). Based on the present observations indicating a correlation between PARP-1 and VEGF-A in human epithelial ovarian cancer, we hypothesized that PARP-1 promotes angiogenesis by upregulating VEGF-A. In confirmation of this hypothesis, knockdown of PARP-1 significantly decreased the expression and secretion of VEGF-A in SKOV3 cells. These results are consistent with a study by Rajesh et al (5), who demonstrated that the PARP inhibitors 3-AB and PJ34 inhibited the VEGF-induced proliferation, migration and angiogenic capacity of HUVECs. However, the underlying mechanism by which PARP-1 directly or indirectly regulates VEGF-A requires further investigation.

In summary, the present study suggests that PARP-1 is overexpressed and promotes angiogenesis in epithelial ovarian cancer by regulating VEGF-A. As it is overexpressed in ovarian cancer and is important in tumorigenesis and angiogenesis, PARP-1 may represent a potential therapeutic target for ovarian cancer.

\section{Acknowledgements}

This study was supported by grants from the National Natural Science Foundation of China (grant no. 81441075) and the Natural Science Foundation of Shandong Province (grant nos. ZR2014HM108 and ZR2013HQ030).

\section{References}

1. Desai A, Xu J, Aysola K, Qin Y, Okoli C, Hariprasad R, Chinemerem U, Gates C, Reddy A, Danner O, et al: Epithelial ovarian cancer: An overview. World J Transl Med 3: 1-8, 2014.

2. Itamochi H: Targeted therapies in epithelial ovarian cancer: Molecular mechanisms of action. World J Biol Chem 1: 209-220, 2010

3. Schiavone MB, Bashir S and Herzog TJ: Biologic therapies and personalized medicine in gynecologic malignancies. Obstet Gynecol Clin North Am 39: 131-144, 2012.

4. Ocana A, Pandiella A, Siu LL and Tannock IF: Preclinical development of molecular-targeted agents for cancer. Nat Rev Clin Oncol 8: 200-209, 2010.

5. Rajesh M, Mukhopadhyay P, Godlewski G, Bátkai S, Haskó G, Liaudet L and Pacher P: Poly(ADP-ribose)polymerase inhibition decreases angiogenesis. Biochem Biophys Res Commun 350: 1056-1062, 2006.

6. Langelier MF and Pascal JM: PARP-1 mechanism for coupling DNA damage detection to poly(ADP-ribose) synthesis. Curr Opin Stru Biol 23: 134-143, 2013.

7. Horton JK, Stefanick DF and Wilson SH: Involvement of poly(ADP-ribose) polymerase activity in regulating Chk1-dependent apoptptic cell death. DNA Repair (Amst) 4: 1111-1120, 2005.

8. Shunkwiler L, Ferris G and Kunos C: Inhibition of poly(ADP-Ribose) polymerase enhances radiochemosensitivity in cancers proficient in DNA double-strand break repair. Int J Mol Sci 14: 3773-3785, 2013.

9. Krietsch J, Caron MC, Gagné JP, Ethier C, Vignard J, Vincent M, Rouleau M, Hendzel MJ, Poirier GG and Masson JY: PARP activation regulates the RNA-binding protein NONO in the DNA damage response to DNA double-strand breaks. Nucleic Acids Res 40: 10287-10301, 2012.

10. Zhang J, Kan Y, Tian Y, Wang Z and Zhang J: Effects of poly (ADP-ribosyl) polymerase (PARP) inhibitor on cisplatin resistance \& proliferation of the ovarian cancer $\mathrm{C} 13^{*}$ cells. Indian J Med Res 137: 527-532, 2013.

11. Pyriochou A, Olah G, Deitch EA, Szabó C and Papapetropoulos A: Inhibition of angiogenesis by the poly(ADP-ribose) polymerase inhibitor PJ-34. Int J Mol Med 22: 113-118, 2008.

12. Livak KJ and Schmittgen TD: Analysis of relative gene expression data using real-time quantitative PCR and the 2(-Delta Delta C(T)) Method. Methods 25: 402-408, 2001.

13. Gonçalves A, Sabatier R, Charafe-Jauffret E, Gilabert M, Provansal M, Tarpin C, Extra JM, Viens P and Bertucci F: Triple-negative breast cancer: Histoclinical and molecular features, therapeutic management and perspectives. Bull Cancer 100: 453-464, 2013 (In French).

14. Salemi M, Galia A, Fraggetta F, La Corte C, Pepe P, La Vignera S, Improta G, Bosco P and Calogero AE: Poly (ADP-ribose) polymerases 1 protein expression in normal and neoplastic prostatic tissue. Eur J Histochem 57: e13, 2013.

15. Porcelli L, Quatrale AE, Mantuano P, Leo MG, Silvestris N, Rolland JF, Carioggia E, Lioce M, Paradiso A and Azzariti A: Optimize radiochemotherapy in pancreatic cancer: PARP inhibitors a new therapeutic opportunity. Mol Oncol 7: 308-322, 2013.

16. Kim J, Pyun JA, Cho SW, Lee K and Kwack K: Lymph node metastasis of gastric cancer is associated with the interaction between poly (ADP-ribose) polymerase 1 and matrix metallopeptidase 2. DNA Cell Biol 30: 1011-1017, 2011.

17. Rojo F, García-Parra J, Zazo S, Tusquets I, Ferrer-Lozano J, Menendez S, Eroles P, Chamizo C, Servitja S, Ramírez-Merino N, et al: Nuclear PARP-1 protein overexpression is associated with poor overall survival in early breast cancer. Ann Oncol 23: 1156-1164, 2012.

18. Ledermann J, Harter P, Gourley C, Friedlander M, Vergote I, Rustin G, Scott CL, Meier W, Shapira-Frommer R, Safra T, et al: Olaparib maintenance therapy in patients with platinum-sensitive relapsed serous ovarian cancer: A preplanned retrospective analysis of outcomes by BRCA status in a randomised phase 2 trial. Lancet Oncol 15: 852-861, 2014.

19. Choi JY, Jang KT, Shim YM, Kim K, Ahn G, Lee KH, Choi Y, Choe YS and Kim BT: Prognostic significance of vascular endothelial growth factor expression and microvessel density in esophageal squamous cell carcinoma: Comparison with positron emission tomography. Ann Surg Oncol 13: 1054-1062, 2006.

20. Rau KM, Huang CC, Chiu TJ, Chen YY, Lu CC, Liu CT, Pei SN and Wei YC: Neovascularization evaluated by CD105 correlates well with prognostic factors in breast cancers. Exp Ther Med 4: 231-236, 2012. 
21. Wang L, Liu X, Wang $\mathrm{H}$ and Wang S: Correlation of the expression of vascular endothelial growth factor and its receptors with microvessel density in ovarian cancer. Oncol Lett 6: 175-180, 2013.

22. Li Q, Li M, Wang YL, Fauzee NJ, Yang Y, Pan J, Yang L and Lazar A: RNA interference of PARG could inhibit the metastatic potency of colon carcinoma cells via PI3-kinase/Akt pathway. Cell Physiol Biochem 29: 361-372, 2012.
23. Quiles-Perez R, Muñoz-Gámez JA, Ruiz-Extremera A, O'Valle F, Sanjuán-Nuñez L, Martín-Alvarez AB, Martín-Oliva D, Caballero T, Muñoz de Rueda P, León J, et al: Inhibition of poly adenosine diphosphate-ribose polymerase decreases hepatocellular carcinoma growth by modulation of tumor-related gene expression. Hepatology 51: 255-266, 2010.

24. Claesson-Welsh L and Welsh M: VEGFA and tumor angiogenesis. J Intern Med 273: 114-127, 2013. 